\title{
The effect of the exercises brain on boxers' eye-hand coordination, dynamic balance and visual attention performance
}

\author{
Çetin O. ${ }^{\mathrm{ABCDE}}$, Beyleroğlu M. ${ }^{\mathrm{ABCDE}}$, Bağ1ş Y.E. ${ }^{\mathrm{ACDE}}$, Suna G. ${ }^{\mathrm{ACDE}}$ \\ Educational Science Institute, Physical Education and Sports Education Department, Sakarya University, Turkey
}

Authors' Contribution: A - Study design; B - Data collection; C - Statistical analysis; D - Manuscript Preparation; E - Funds Collection.

\begin{abstract}
Purpose: $\quad$ The purpose of this study is to investigate whether exercising brain performed by boxers have any kind of effect on visual attention, eye-hand coordination and dynamic balance performances of athletes.

Material: $\quad$ In accordance with the purpose of this study, 29 athletes who do exercises regularly at Sakarya Boxing Club have been divided into two groups as control and experiment group. The Star Excursion Balance test was used to measure participants' dynamic balance performance, the Bourdon-Wiersma Stipple test was used to measure visual attention, and Alternate Hand Wall Toss test was used to measure eye-hand coordination as pre and posttest. Repeated Measures Anova analysis was used for analysis of the obtained data.

Results: In terms of the obtained data, it has been observed that between pre and pro tests a significant development pattern shows itself for whole study group. On the other hand, as for the investigation of the pre and post-test interactions of experiment and control group, When pre and post-test interactions of experimental and control groups were examined, there was no significant difference in visual attention and dynamic balance development, but in eyehand coordination test, improvement in experimental group was found to be higher than in control group.

Conclusions: The findings of the study in which exercising brain applied to boxers for 8 weeks showed that there was no significant difference between dynamic balance and visual attention development values, but there was a significant difference between control and experimental groups, eye-hand coordination development values.

Keywords: cognitive exercise, dynamic balance, visual attention, hand-eye coordination.
\end{abstract}

\section{Introduction}

Today's sport has started to be perceived and practiced very differently than in the recent past. Sportive activities that are increasingly different have been done for different purposes [1]. One of the sportive activities that differentiate is brain exercises. Life kitetic found and developed by Horst Lutz et al; is a brain training system that is practiced using exercises combined with visual, mental and cognitive tasks. These exercises have such physical and cognitive positive effects as concentration, problem solving skills, reflexes, balance, coordination and coping with stress in elite athletes and sedentary people [2]. Visual attention, hand-eye coordination and balance have been included in the literature from past to present in terms of general knowledge, especially in research articles and the effect of various exercises on these characteristics have been investigated $[3,4,5]$. However, whether brain exercise has an impact on these physical and cognitive features is a problem that needs to be investigated.

Attention is considered to be the subjective awareness of the world around us at first, while attention is considered as a neural system in which visual, auditory, and motor systems are used for the selection of similar information by the neuropsychological approach [6]. Visual attention refers to a series of cognitive processes that allow the necessary information to be found from scattered (complex) visual scenes, and the unnecessary information to be filtered out [7]. In the literature, there are studies examining the cognitive features such as visual attention and the effects of exercises on cognitive performance to improve the performance of these features. In the present study, the effects of acute exercise (c) Çetin O., Beyleroğlu M., Bağış Y.E., Suna G., 2018 doi:10.15561/20755279.2018.0301 on cognitive performance were investigated and specific exercises were reported to have a greater effect than those of acute exercise [8]. When we look at the specific exercise studies on visual attention, it is concluded that visual attention can be improved and trained by specific web based training [9].

Hand-eye coordination is a visual system capability that coordinates the revised information to control hands, directing and managing tasks such as writing or capturing a ball successfully [10].

When the researches carried out are examined, it is concluded that acute and specific exercises have positive effects on hand-eye coordination. In the related study on the effects of hand-eye coordination on balance and handeye coordination in the elderly, swimming exercises was proven to have positive effects on hand-eye coordination [11]. In another study on hand-eye coordination training of table tennis athletes, it is reported that the table tennis athletes trained on hand-eye coordination performed better than those who were not trained [12]. In this study, we investigated the effects of hand-eye coordination on the effects of Traditional Chinese coordination exercises, TaiChiChuan (TCC), on hand-eye coordination in the elderly and it is reported that those who applied handeye coordination exercises exhibited better hand-eye coordination performance than those who did not [13].

Balance is defined as the state or ability to maintain control over the body's support centre. The most important feedback mechanisms in balance are vestibular, somatosensory and visual systems $[6,14]$. In this study, we will discuss the relationship between the equilibrium (balance) and the relationship between the two variables $[15,16]$. When we examine the effects of various exercise programs on balance development, it is seen that exercise 
programs have positive effects on balance development. The effects of pliometrics, dynamic stabilization and balance training for 7 weeks on the strength, balance and fall forces of female athletes were examined and it is reported that these exercises have a positive effect on balance [17]. Similar results were obtained in the study of different exercise methods on the follow-up of balance development. Especially in athletes, it is reported that neuromuscular training or specific exercises such as yoga for 6 to 8 weeks has a positive effect on the development of balance [18, 19, 20].

Brain exercises were developed by Lutz and his colleagues as a multimodal training method that combines coordinated, cognitive and visual tasks and performs the physical activities of the participants and also cognitive activities. This training method consists of combinations of motor activities, cognitive skills, visual perception training and especially perception of peripheral vision. The main characteristic of this training method is to move the limbs in different combinations, capture, throw, and thus to train visual perception and limb-eye coordination [21].

The effects of acute and specific exercises on cognitive and motor features, especially visual attention, hand-eye coordination and balance were investigated in the investigated studies. However, there was no study that examined the effects of brain exercise on these three abilities as a whole. Therefore, this research will help us to understand whether the brain exercises that are increasingly being applied by athletes and sedentary people have effects on visual attention, hand - eye coordination and balance ability. When we consider the concept above, it may be stated that the aim of this study is to investigate whether brain exercises in boxers for 8 weeks have effects on athletes' visual attention, hand-eye coordination and balance performances.

\section{Material and Methods}

\section{Participants}

29 athletes who were trained regularly in Sakarya Boxing Club participated voluntarily in this study using experimental pattern with control group. The participants were randomly divided into two groups. While 15 of them were in the experimental group, 14 of them were included in the control group.

Physical characteristics of the participants; age is $20.4 \pm 0.8$ years, body weight is $74 \pm 9.4 \mathrm{~kg}$ and height is $176 \pm 8.1 \mathrm{~cm}$.

\section{Data Collection Tools}

The body weights of the athletes were measured with Seca 808 (Germany) brand electronic scales with $0.1 \mathrm{~kg}$ sensitivity and height measurements with portable stadiometer with Seca 213 (Germany) brand with $1 \mathrm{~mm}$ sensitivity. In the application of the Visual attention test, the Bourdon attention test was used for marking papers belonging to the Wiersma form with 450 figures on it. The Dunlop brand tennis ball and the Casio HS-80TWDF brand chronometer were used in the application of the Alternate Wall Hand Toss for hand-eye coordination. In the implementation of the Star Excursion dynamic balance test, the platform prepared in accordance with the test protocol was prepared and Troy 23124 brand strip meters were used to measure the reach distances of the participants.

\section{Collection of Data}

Anthropometric measurements, balance and hand-eye coordination tests were carried out in Sakarya University Faculty of Sports Sciences gymnasium saloon and visual attention test was carried out in the class which was arranged for testing. The anthropometric measurements, balance and attention tests of the control and experiment groups were carried out on the same day and hand-eye coordination was carried out on the same day. Following the pre-test, brain exercises were performed for 8 weeks and 3 days a week in addition to the branch training of experimental group. The control group was not included in the brain exercise and they continued their own branch training with the experimental group. At the end of 8 weeks, the participants underwent the final tests within the framework of the selected tests protocols.

Determination of dynamic balance performance

The Star Excursion Balance Test (SEBT) was used to determine the dynamic balance and dynamic postural control performances of the participants. SEBT is a balance test that requires force, flexibility, and proprioception performance used in dynamic balance measurement [22]. The person who performs the test tries to reach out in 8 different directions with one leg and 45 degrees of angle between them, while the other leg remains in the centre point and tries to maintain its stability [23].

The validity of the SEBT test was reported between 0.89 and 0.92 [24]. After warming the participants for general and test, they reached 8 different directions on the test platform three times, respectively, and recorded in meters and centimeters, which are best from 3 accesses. The same practice was carried out for the other foot. In order to ensure the validity of the test, leg-length measurements were taken for each of the participants. The measurement was repeated when participants moved the balance leg from the platform centre, lost balance or were unable to contact the stated directions.

\section{Measurement of visual attention performance}

In order to measure the visual attention performance of the participants, the Bourdon-Wiersma stipple test developed and then conducted by Benjamin Bourdon was used. In this test, participants are asked to find and select the necessary information from complex visual scenes in accordance with visual attention. The Bourdon-Wiersma test form contains three, four and five point shapes and the participants are requested to mark four point ones of these 3 figures [25, 26] Participants were given 6 minutes to mark the correct shapes on the form with 450 figures and the correct and wrong shapes were recorded.

Measurement of hand-eye coordination performance In order to determine the hand-eye coordination 


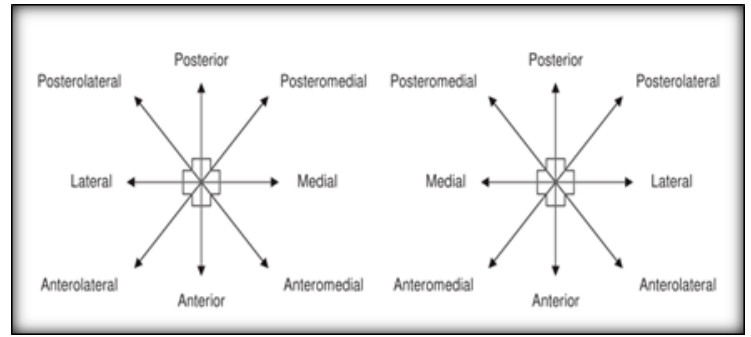

Figure 1. Reaching directions of Star Excursion Balance Test

performance of the participants, the Alternate Hand Wall Toss test was used. The number of successful catches carried out in the test aimed at capturing the tennis ball with the right hand and the left hand is recorded in the 30-minute period of time [27].

Data Analysis

SPSS 22 program was used in the analysis of the obtained data. ANOVA (Repeated measurements ANOVA) analysis was used to determine whether the change in the pre and final test measurements of the participants in the experiment and control group was significantly different from the groups. $\mathrm{P}<0.05$ was considered as significant.

\section{Results}

There was a significant improvement in hand-eye coordination between the pre-test and the final test in the whole research group $[F(1.27)=70.493 ; p<0.05]$. When the experimental-control group's pre-test-final test interactions were examined, it is seen that the development of the experimental group was significantly higher than the control group $[\mathrm{F}(1.27)=8.439 ; \mathrm{p}<0.05]$.

There is a significant improvement in the attention skill among the pre-test and final test in the whole Research Group $[F(1.27)=6.495 ; p<0.05]$. When the prepost test interactions of the experiment-control group are examined, it was seen that the experiment-control group showed a development at a similar level and the development of attention level was not statistically different in terms of the experiment-control group $[\mathrm{F}(1.27)=.014 ; \mathrm{p}>0.05]$.

There is a significant improvement in the balance skill between pre-test and final test in the whole research group

Table 1. Comparison of Hand-Eye Coordination of Pre and Post Test Results of Boxers

\begin{tabular}{llccc}
\hline Tests & Groups & $\mathbf{N}$ & $\mathbf{X}$ & Ss \\
\hline Pre-test & Experiment & 15 & 20.73 & 5.74 \\
& Control & 14 & 18.71 & 5.12 \\
Post-test & Experiment & 15 & 26.47 & 5.20 \\
\hline
\end{tabular}

Table 2. Comparison of Visual Attention of Pre and Post Test Results of Boxers

\begin{tabular}{llccc}
\hline Tests & Groups & $\mathbf{N}$ & $\mathbf{X}$ & Ss \\
\hline & Experiment & 15 & 72.87 & 11.13 \\
Pre-test & Control & 14 & 66.50 & 9.76 \\
Post-test & Experiment & 15 & 78.13 & 12.88 \\
\hline
\end{tabular}


Table 3. Comparison of the Dynamic Balance of Pre and Post Test Results of Boxers

\begin{tabular}{lllll}
\hline Tests & Groups & $\mathbf{N}$ & $\mathbf{X}$ & Ss \\
\hline & Experiment & 15 & 86.01 & 7.906 \\
Pre-test & Control & 14 & 90.16 & 4.304 \\
Post-test & Experiment & 15 & 87.92 & 8.992 \\
& Control & 14 & 91.39 & 5.065 \\
\hline
\end{tabular}

$[\mathrm{F}(1.27)=6.104 ; \mathrm{p}<0.05]$. Experimental-control group's pre-and final-test interactions examined have shown similar levels of improvement in the experimental and control groups and it is concluded that the experimental and control groups are statistically significantly different at the level of the balance of the development $[\mathrm{F}(1.27)=$ .292; $\mathrm{p}>0.05]$.

\section{Discussion}

Boxing is a combination of technical, tactical, durability, speed and coordination features. In addition to the basic characteristics of the fist, others are also part of the ring. Success will be achieved with the completion of these features and the proliferation of scientific research, development of general training programs, physical characteristics and the selection of talented athletes will be possible by teaching of technical and tactical studies through different methods.

One of the most important findings of this study is that the effects of brain exercise on the various coordinative and cognitive characteristics of athletes; hand-eye coordination repeated measurements on the preliminary and final test results were found to be significantly higher in the ANOVA analysis than in the experimental group $[\mathrm{F}(1,27)=8,439 ; \mathrm{p}<0.05]$. The most obvious characteristics of brain exercises are moving the limbs in different combinations, capturing, throwing objects, and thus training visual perception and limbeye coordination [21]. Therefore, visual skill training is practiced intensively in brain exercises. When the literature is examined, it has been observed that all exercises including brain exercises and similar visual training have positive effects on hand-eye coordination and this finding supports the results in our study. When we look at studies involving visual training like brain exercise, it is seen that Du Toit and others (2011) reported that visual training made significant progress in hand-eye coordination of university students. In another study, "Eyerobic" (visual skill training) exercises were applied to female footballers and at the end of the exercise period, balance and hand-eye coordination values were reported to be higher than control group [28].
It is known that balance skill, which is the basis of performance, plays an important role in the successful display of many sports skills, in changing directions, in stopping, in starting, keeping the object, in moving the object, and in maintaining the body's specific position.

According to Meinel and Schnabel, balance ability is defined as keeping the whole body in balance and maintaining the state of the body during and after displacement of the body [29]. Human's ability to maintain balance can be defined as a determining factor in the development of other motor systems [30]. The control of balance is a complex motor capability that includes the integration of sensory inputs as well as the planning and application of flexible motion patterns [31]. The ability of the whole body to move in coordination as a whole takes place in a correct proportion with the ability to balance [32].

In this study, based on the results of the measurements on dynamic balance, the development differences in balance level are not statistically significant in terms of experiment and control group. $[\mathrm{F}(1,27)=, 292 ; \mathrm{p}>0.05]$. In a study conducted on basketball players, it was reported that brain exercise training for 12 weeks at basketball players had no effect on balance scores of the athletes [33]. Similarly, at the study conducted on young football players, it was reported that there was no significant difference between the pre-test and final test scores of the participants in the brain exercise training for 8 weeks [34]. The researches examining the effects of brain exercise on balance support the findings of this study.

In this study, the measurements of visual attention were examined and it was concluded that the development of the control and experiment group showed similar development but the visual attention level was not significantly different from the experimental and control group $[F(1,27)=, 014 ; p>0,05]$. The resources that scientists who develop and practice brain exercises refer to as a reference to prove the effects of these exercises on important cognitive skills and visual attention are generally about young children or students who have difficulty reading [34]. There are studies in the literature that reported positive effects of specific exercises on 
visual attention [8]. In addition to this study, there was only one study which examined the development of visual attention of athletes who train intensively in brain exercise and contrary to this research, positive results were reported. In this research conducted by Vural (2016), it was found that brain exercises had positive effects on the visual attention performances of athletes. Therefore, more research is needed to examine the impact of brain exercise on visual attention development, especially in trained and advanced athletes.

As a result, the results of this study showed that there was a significant difference in hand eye coordination development values between control and experimental groups of brain exercises performed in boxers for 8 weeks, while dynamic balance and visual attention did not differ significantly between development values. Since brain exercise exercises are based on simultaneous perception, evaluation, making the right choice and making the appropriate decision, we believe that coaches should be involved in brain exercise exercises in athletes 'training and that the criteria for athletes' performance will contribute significantly to the field of sports sciences as they have a significant place in determining the current levels of athletes.

\section{Conclusions}

We also think that brain exercise will help athletes make the right decisions in training or competition by enabling them to think continuously. It is thought that after the exercises that are performed in this way, the athlete will soon be able to demonstrate the dominance of the more advanced coordinative features and will become a well-featured athlete who understands fast and makes decisions quickly by reflecting them to his team or to himself. Because of such many benefits, we think that sports scientists and coaches will benefit from brain exercises and it will be important to include brain exercises in training plans.

\section{Conflict of interests}

The authors state that there is no conflict of interest. 


\section{References}

1. Çeliksoy MA, Kaya T, Köse S. Development of Sports Sciences, Relation With Education of Physical Education and Sports. 77th International Sports Science Congress, Alanya; 2002. P. 120-125. (in Turkish)

2. Braun A. A work out for the brain. FIFA 1904 Published monthly by the Federation Inernationale De Football Association (FIFA); 2016

3. Udermann BE, Murray SR, Mayer JM, Sagendorf K. Influence of cup stacking on hand-eye coordination and reaction time of second grade students. Perceptual and motor skills, 2004; 98(2):409-14.

4. Hardy JL, Drescher D, Sarkar K, Kellet G, Scanlon M. Enhancing visual attention and working memory with a Web-based cognitive training program. Mensa Research Journal, 2011; 42 (2):13-21.

5. Mohammadi V, Alizadeh M, Gaieni A. The effects of six week strength exercises on static and dynamic balance of young male athletes. Procedia - Social and Behavioral Sciences, 2012; 31:247-50.

6. Posner MI, Grossenbacher PG, Comton PE. The Neuropsychology of High-level Vision: Collected Tutorial Essays (Carnegie Mellon Symposia on Cognition Series). New Jersey Lawrance Erlbaum Associates, Inc.; 2011.

7. McMains Stephanie A, Sabine Kastner. Visual attention. Encyclopedia of Neuroscience. Springer Berlin Heidelberg; 2009.

8. Chang YK, Labban JD, Gapin JI, Etnier JL. The effects of acute exercises on cognitive performance: a meta-analysis. Brain research, 2012; 1453: 87-101.

9. Scanlon M, Drescher D, Sarkar, K. Improvement of visual attention and working memory through a web-based cognitive training program. A Lumos Labs White Paper; 2007.

10. Natarajan P, Malliga TJ. Hand to eye co-ordiination error assessment using simple maze test. International Journal of Engineering Science and Technology, 2011; 3(3): 1967-71.

11.Hsu HC, Chou SW, Chen CPC, Wong AMK, Chen CK, Hong JP. Effects of swimming on eye hand coordination and balance in the elderly. The journal of nutrition, health \& aging, 2010; 14(8): 692-695.

12. Paul M, Biswas SK, Sandhu JS. Role of sports vision and eye hand coordination training in performance of table tennis players. Brazilian Journal of Biomotricity, 2011; 5(2):106-16.

13. Pei YC, Chou SW, Lin PS, Lin YC, Hsu TH, Wong AM. Eye-hand coordination of elderly people who practice Tai Chi Chuan. Journal of the Formosan Medical Association, 2008; 107(2): 103-110.

14. Yildizer G. Effects of 8-week Core Stability Training on Junior Male Soccer Players'Static Balance Performance. Graduate Thesis. Ankara: Middle East Technical University, Institute of Social Sciences, 2014.

15. Ak E. Investigation Of The Use Of The Arms In
Recovering From Postural Perturbations. Doctoral Thesis. Ankara: Middle East Technical University Institute of Social Sciences; 2014. (in Turkish)

16. Lesinski M, Hortobágyi $\mathrm{T}$, Muehlbauer $\mathrm{T}$, Gollhofer A, Granacher U. Effects of balance training on balance performance in healthy older adults: a systematic review and meta-analysis. Sports medicine, 2015; 45(12):1721-1738.

17. Myer GD, Ford KR, Brent JL, Hewett TE. The effects of plyometric vs. dynamic stabilization and balance training on power, balance, and landing force in female athletes. The Journal of Strength \& Conditioning Research, 2006; 20(2): 345-353.

18. McLeod TC, Armstrong T, Miller M, Sauers JL. Balance improvements in female high school basketball players after a 6-week neuromuscular-training program. J Sport Rehabil, 2009;18(4):465-481.

19. Filipa A, Byrnes R, Paterno MV, Myer GD, Hewett TE. Neuromuscular training improves performance on the star excursion balance test in young female athletes. Journal of orthopaedic \& sports physical therapy, 2010;40(9):551-558.

20. Kasse CA, Prado ET, Raso V, Scharlach RC. Hatha yoga on body balance. International Journal of Yoga, 2014; 7(2):133-137.

21. Demirakca T, Cardinale V, Dehn S, Ruf M, Ende G. The Exercising Brain: Changes in Functional Connectivity Induced by an Integrated Multimodal Cognitive and Whole-Body Coordination Training. Neural Plasticity, 2016;2016:1-11. doi:10.1155/2016/8240894

22. Kinzey SJ, Armstrong CW. The reliability of the starexcursion test in assessing dynamic balance. Journal of Orthopaedic \& Sports Physical Therapy, 1998;27(5): 356360.

23. Olmsted LC, Carcia CR, Hertel J, Shultz SJ. Efficacy of the star excursion balance tests in detecting reach deficits in subjects with chronic ankle instability. Journal of athletic training, 2002; 37(4): 501-508.

24. Gribble PA, Kelly SE, Refshauge KM, Hiller CE. Interrater reliability of the star excursion balance test. Journal of athletic training, 2013; 48(5):621-626.

25. Kaymak S. The Effect of the Attention Collecting Education Program on the Development of Attention Collecting Skills of Primary and Secondary Grade Students. Unpublished Doctorate Thesis, Ankara: University Institute of Social Sciences; 2003. (in Turkish)

26. Bozan A, Akay Y. The Effect of Attention Improvement Training on the Primary School 5th Grade Students' Skills of Gathering Attention. Western Anatolia Journal of Educational Science, 2012; 3(6):53-66. (in Turkish).

27. Du Toit PJ, Kruger PE, Mahomed AF, Kleynhans M, Jaydu Preez T, Govender C, Mercier J. The effect of sports vision exercises on the visual skills of university students: sport science. African Journal for Physical Health Education, Recreation and Dance, 2011; 17(3):429-440.

28. McLeod B. Effects of Eyerobics visual skills training on 
selected performance measures of female varsity soccer players. Perceptual and motor skills, 1991;72(3):863-866.

29. Altay F. Biomechanics analysis of the side balance activity after chaine rotation under the two varying speeds in the rytmic gymnastics. [PhD Thesis]. Ankara: Hacettepe University Health Sciences Institute; 2001. (in Turkish).

30. Erkmen N, Suveren S, Göktepe AS, Yazıcıoğlu K. The Comparison of Balance Performance of the Athletes Who are in Different Branches. Spormeter, 2007; 3: 115-122. (in Turkish)
31. Hrysomallis C. Balance ability and athletic performance, Sports Med, 2011; (3)41: 221-232.

32. Taşkın C, Karakoç Ö, Yüksek S. Investigating Static Balance Performance Status of Deaf Handicapped Male Volleyball and Handball Players. ASOS, 2015; 17:248-55. (in Turkish)

33. Vural M.U. Effects of life kinetik training on balance, reaction time and attention on young male basketball players. Graduate Thesis. Ankara: Gazi University Institute of Health Sciences; 2016. (in Turkish)

34. Peker AT. The effect of life kinetic trainings on coordinative abilities, Graduate Thesis. Konya: Selcuk University Institute of Health Sciences; 2014.. (in Turkish). 
Information about the authors:

Çetin O.; http://orcid.org/0000-0001-6841-5518; Educational Science Institute, Physical Education and Sports Education Department, Sakarya University, 54050, Turkey.

Beyleroğlu M.; http://orcid.org/0000-0002-2223-0064; Educational Science Institute, Physical Education and Sports Education Department, Sakarya University, 54050, Turkey.

Bağış Y.E.; http://orcid.org/0000-0003-3170-7343; Educational Science Institute, Physical Education and Sports Education Department, Sakarya University, 54050, Turkey.

Suna G.; (Corresponding author); http://orcid.org/0000-0002-2125-9105; gurhan suna@windowslive.com; Educational Science Institute, Physical Education and Sports Education Department, Sakarya University, 54050, Turkey.

Cite this article as: Cetin O, Beyleroğlu M, Bağ 1 Ș YE, Suna G. The effect of the exercises brain on boxers' eye-hand coordination, dynamic balance and visual attention performance. Physical education of students, 2018;22(3):112-119. doi: $10.15561 / 20755279.2018 .0301$

The electronic version of this article is the complete one and can be found online at: http://www.sportedu.org.ua/index.php/PES/issue/archive

This is an Open Access article distributed under the terms of the Creative Commons Attribution License, which permits unrestricted use, distribution, and reproduction in any medium, provided the original work is properly cited (http://creativecommons.org/licenses/by/4.0/deed.en).

Received: 06.05.2018

Accepted: 02.06.2018; Published: 27.06.2018 